\title{
Nanoporous plasmonic coatings
}

\author{
A. I. Maaroof, A.R. Gentle, M B. Cortie, G. B. Smith* \\ Institute of Nanoscale Technology, University of Technology, Sydney \\ PO Box 123, Broadway, NSW, 2007, Australia
}

\begin{abstract}
The electrical and optical properties of mesoporous gold are compared to those of thin porous gold films and a simulated thin film made by randomly distributing voids in gold, until the voids fill $76 \%$ of film volume. All layers are electrically conducting but in some cases the critical percolation thresholds are close to zero, so conduction is possible at very high void content. Significant qualitative differences are apparent between the properties of mesoporous gold, and very thin sputtered gold containing voids, in plasmonic responses at optical frequencies and in dc resistance, both as a function of fill factor. The mesoporous films have an effective plasma frequency determined by void fill factor and structure, but do not support surface plasmons. In contrast thin porous gold layers display optical features associated with localized and de-localized surface plasmons. Sputtered porous gold is 2-dimensional and its percolation threshold requires a "Swisscheese" rather than particle cluster model. Thicker mesoporous layers have critical parameters consistent with very high connectivity, or equivalently large hyper-dimensionality. Our meso-gold samples display various hyper-dimensionalities from 3 to above 10 .
\end{abstract}

Keywords: nanoporous metal, thin film, effective medium, plasma frequency, hyper-dimensions, percolation

\section{INTRODUCTION}

\subsection{Structural issues}

Coatings which both conduct and are highly porous can yield a wide variety of optical and electrical responses as both the void fraction and the structure vary. Conducting structures in which the components do not have long range order and the non-conducting component makes up a large percentage of the volume, in the range $35 \%$ to $90 \%$, are our main concern here. Topology and effective 'dimensionality' $\mathrm{d}$ in some nanoporous structures can be controlled and varied widely by altering growth conditions and growth techniques. Hyper-dimension $\mathrm{d}$ is determined by the connectivity $\mathrm{z}$ of the structure, such that $\mathrm{z} / 2=\mathrm{d}$. We use the term 'geometry' to describe the spatial dimension $\mathrm{D}$ of the solid. Some porous coating have $\mathrm{D}=2$ and some have $\mathrm{D}=3$. For conduction to occur percolation of the metal component must be possible. If the voids are sufficiently small optical scattering is not important and we find specular behavior. In this case standard spectrophotometers and ellipsometers can be used to examine spectral reflectance, transmittance and absorption, as well as to extract effective homogeneous optical constants. These homogenized optical responses arise from far-field data, which involves averages over near-field responses. In recent years more insights into the near-field have become available from NSOM experiments, and computer simulations of near-field intensities. The apparent dimensionality should impact on both near- and far-field optical results and on resistance.

\subsection{Electrical and plasmonic responses in porous networks}

DC electrical properties are quite sensitive to void content and structure. Studies in this area have been largely based on percolation theory for spherical or circular particle arrays and experimental analysis has been mainly with 2-dimensional models of very thin metal films which are not fully dense. We compare optical and electrical properties on similar structures grown in our laboratory with mesoporous gold which has 3 or higher dimensional character.

*g.smith@uts.edu.au; Phone: +61 29514 2224; Fax: +61 295142219 
Further insights are gained by looking at models of gold containing a random distribution of voids within its volume, with the void diameter smaller than layer thickness to ensure at least 3 - dimensionality. Our mesoporous conducting networks, while obviously above the percolation threshold can still be characterized by a critical percolation volume fraction of metal $f_{\mathrm{c}}$. The parameter $f_{\mathrm{c}}$ is very small, as low as 0.07 , in our samples, well below observed 2-D values to date which are typically in the range 0.3 to $0.5[1,2]$. Recently these 2-D percolation models have been adapted to model 2-D optical properties, in particular plasmonic responses, providing a link between dc and optical responses [3]. Two classes of surface plasmons arise in these models, localized and delocalized. We shall show that, as more mass of conductor is present in our meso-layers, topology evolves, along with the percolation threshold and critical parameters. Their bulk and surface plasmon character also evolves, as does de conductivity. It was a surprise to find a class of nanostructured metal films with very high void content which also had the simplest possible effective plasmonic response, namely that of a simple dense metal. This is evidenced by their Drude character at longer wavelengths $[4,5]$.

Three distinct classes of random nanoporous conductors are compared here in terms of plasmonic behavior. The first type, granular thin films, is well known and much data exists in both far- and near-field regimes. It involves thin noble metal films whose growth has been stopped before or after percolation, and before they become fully dense [6] so they are quite thin, typically less than $12 \mathrm{~nm}$ thick. The voids in such films traverse the film from top to bottom. Far-field data is roughly metal-like, except for a broad plasmon resonant feature not found in dense metals, which in gold and silver appears in the long-wavelength visible or near-infrared (NIR). Such data cannot be modeled with standard effective medium approaches because surface plasmon polaritons coupled through the voids are involved [7]. Near-field data and models $[8,9]$ show very complex and varied local resonance effects as both position in the nanostructure and wavelength shift, but spatial averaging as found in far-field data seems to wash out localised sharp spectral features.

The second type has two sub-classes. First metal colloidal structures, which involve clusters of touching metal nanoparticles . Limited data is available on large percolating arrays, though interesting studies exist on some percolating fractal structures including those formed from carbon nanoparticles in space. Much computer modeling has been carried out on clusters of spherical gold and silver nanospheres, though cluster size has been limited by computer time. Sixparticle clusters of gold display some residual multiple resonant spectral features [10]. Less has been done with the second class or "Swiss-cheese" models where random holes are implanted into the metal. It is known that circular voids [11] in metal can give different percolation limits to arrays of metal circles. We find the Swiss-cheese model applies in very thin gold. When we use "Swiss-cheese" models in three dimensions simulated optical behavior resembles that in experimental samples of mesoporous gold. This simulated optical data, which we present later, is novel and varies smoothly with wavelength in the NIR.

The third type of porous conductor is represented by mesoporous gold. These display the simplest possible plasmonic optical response in that data can be fitted at longer wavelengths with a Drude model $[4,5]$, using a plasma frequency $\omega_{\mathrm{p}}$ which is well below that in dense gold. A reduced $\omega_{\mathrm{p}}$ is expected because of the reduced density of metal and hence free carriers, but the observed changes in $\omega_{\mathrm{p}}$ also depend on structure and this aspect can be attributed to a change in carrier effective mass $m^{*}$ [5]. The $m^{*}$ from optical data can also display critical properties at the percolation threshold which link to dc critical response. The effective medium parameters that describe these structures include void fraction $f$, and one or more structural parameters which we examine later and link to connectivity. Expressions linking these parameters to the observed effective plasma frequency are consistent with data $[4,12]$. Anisotropy in effective plasmonic response can occur in these models. This is a relatively new idea for bulk plasmonic conductors. It is well known in other plasmonic systems, such as gaseous plasmas.

\section{STRUCTURES AND FILM PREPARATION}

\subsection{Thin conducting gold films with voids}

Single layer gold thin films were deposited using dc-magnetron sputtering onto clean optically polished, super-white glass $\left(76 \times 26 \times 1 \mathrm{~mm}^{3}\right)$. The sputtering targets were $99.99 \%$ pure gold discs $(50 \mathrm{~mm}$ diameter), placed $150 \mathrm{~mm}$ away from the substrate. The base pressure was better than $\sim 10^{-6}$ Torr, while sputtering was carried out in presence of flowing $\mathrm{Ar}$, at a pressure of $2 \mathrm{mTorr}$. The resistance of the Au films has been measured in situ similar to the study described by Maaroof and Evans [1]. 


\subsection{Mesoporous gold}

The fabrication process of the mesoporous gold films is similar to that described previously $[4,5,13]$. Briefly, alloy films of $\mathrm{AuAl}_{2}$ were prepared by co-depositing the elements using high vacuum dc magnetron sputtering onto glass substrates. To ensure good homogeneity, uniformity and crystallinity, a rotating stage at $400^{\circ} \mathrm{C}$ substrate temperature was used during deposition. The actual thickness of the final etched films also varies with deposition conditions. After codepositing of $\mathrm{AuAl}_{2}$ on a glass substrate, aluminum was removed from the compound by immersing the films in $\mathrm{NaOH}$ $(0.2 \mathrm{M})$ solution. Scanning electron microscopy (SEM) on Zeiss Supra 55VP was used to study the nanostructure and cross-sections of the films (see [5,13] for example images). Most importantly for linking electrical and optical response and their respective critical parameters, these are 3-dimensional structures. Comparison of these SEM images to those of granular films indicates clearly that the latter are 2-dimensional. This difference is in part due to the wider range of thicknesses available for the mesoporous layers from a few $\mathrm{nm}$ up to 100's of $\mathrm{nm}$ (95 nm maximum was used in this study), while the granular films cease to have voids once thickness is above around 12 to $14 \mathrm{~nm}$ for evaporation and sputtering, and a few $\mathrm{nm}$ with ion-assisted deposition [6]. Their voids traverse the layer from side-to-side fairly directly. Substrate temperature can also influence these limiting thicknesses for noble metal layers. Electrical and optical data (to be presented later) also provides information on dimensionality and its evolution as thickness increases for the same initial set of production conditions.

These networks thus provide a simple practical basis for studying electrical and optical properties in random topologies whose dimensionality and average structure can vary continuously over a very wide range. Since critical behavior depends on dimensionality and geometry, these mesoporous films provide a basis for fundamental experimental insights into critical behavior in hyperdimensions (i.e. under conditions of enhanced connectivity). This dimensionality is controllable. Few practical studies exist on percolation in hyper-dimensional systems though there is a vast theoretical literature. Most experimental work on voided metallic conductors has been limited to the films discussed in section 2.1 and hence to 2-D.

\subsection{Simulated mesoporous gold based on nanoparticle networks}

We have simulated a $100 \mathrm{~nm}$ square portion of a mesoporous gold film to study optical properties using the discrete dipole approximation scattering model DDSCAT [14] for comparison with experimental data and effective medium studies of real films. The target was generated in $15 \mathrm{~nm}$ and $40 \mathrm{~nm}$ thicknesses to match experimental thicknesses, by starting with a 100x100 element block of gold in the appropriate thickness, following by a Monte Carlo-driven hollowing out process in which spherical voids were randomly placed within the block. The voids had a mean diameter of $10 \mathrm{~nm}$ with a standard deviation in diameter of $2 \mathrm{~nm}$ for the $15 \mathrm{~nm}$ thick coating, and 12 and $5 \mathrm{~nm}$ respectively for the $40 \mathrm{~nm}$ thick sample. These dimensions were based on transmission and scanning electron microscope images of voids in a meso-gold sample film. Sufficient voids were implanted in both cases so that the remaining gold made up only $\sim 24 \%$ of the volume of the starting block, which we will show is close to the void content in some of the films produced as described in section 2.2. The section used in simulation is shown in Figure 1, along with the direction of light and the two polarisations used in optical modelling. 


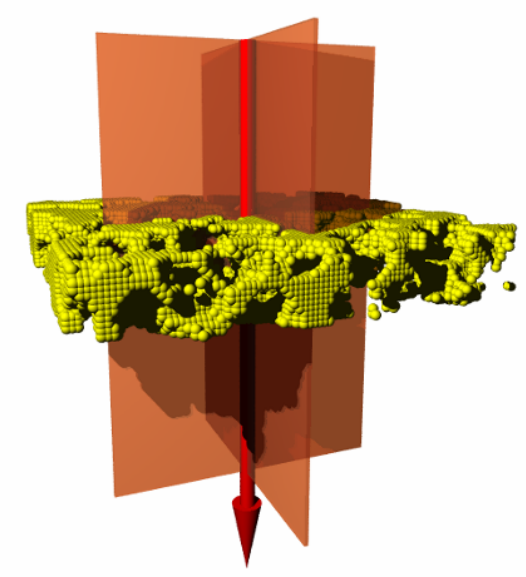

Figure 1: The simulation of mesoporous gold target using "Swiss-cheese" hollowing. The arrow shows light direction and the translucent planes show two polarizations.

\section{RESULTS AND DISCUSSION}

\subsection{Electrical conductivity and structure dependence of percolation}

Resistance data for a sequence of gold thin films sputtered onto glass substrates as a function of mass thickness and decreasing void density, is plotted in Figure 2. A number of publications exist with similar data for silver thin films where $f_{\mathrm{c}} \sim 0.5$ [2], with a small number on gold [15]. In this case results span the percolation threshold which occurs at $f_{\mathrm{Au}}=f_{\mathrm{c}}$. Figure 2 also contains two theoretical plots based on percolation theory. The expression for resistance as a function of metal coverage factor $f$ in equations $(1 \mathrm{a}, \mathrm{b})$ includes both the critical concentration and a critical exponent $\alpha$ [15]. (1a) is for $f>f_{\mathrm{c}}$ and (1b) for $f<f_{\mathrm{c}}$ with $A, B$ constants which determine, $R_{\text {metal }}$ and $R_{\text {dielectric }}$ the limiting values of resistance at $f=1$ and $f=0$ respectively. The $\alpha$ value is linked to dimension with $\alpha=1.3$ for 2-D structures and $\alpha=2$ for 3-D metal structures. Different $\alpha$ values are expected for hyper-dimensional percolation [16].

$R=A\left(f-f_{c}\right)^{-\alpha}, \quad f>f_{c}$,

$R=B\left(f_{c}-f\right)^{\alpha}, \quad f<f_{c}$.

Our data for developing gold thin layers in Figure 2 has $f_{\mathrm{c}}=0.3$ in agreement with previous work on gold [5]. Since it is clearly a 2-D structure from micrographs it seems that the "Swiss-cheese" [11] (hole-in-dense-gold) model is needed for such gold rather than the particle cluster model where $f_{\mathrm{c}}=0.5$, which however works for silver. It seems that $\alpha=2$ gives a much better fit than $\alpha=1.3$. This points to 3-D but critical parameters may also be different in a "Swiss cheese" percolation system. The critical concentration is sensitive to dimension, tending to drop as increasing degrees of freedom or connectivity allow more options for carrier flow. We can use the Bruggeman $(\mathrm{Br})$ effective medium treatment of dc conductivity and optical response for insights into both $f_{\mathrm{c}}$ and effective dimensionality. Connectivity impact is what they have in common. For optical properties we link this aspect to effective depolarisation factors $L$ in a $\mathrm{Br}$ effective medium model. The critical concentration using the Br model is simple, and is given in equation (2) [5]. In an optical Maxwell Garnett (or static Clausius-Mosotti) topology however percolation is always present down to $f=0$ [17] which then becomes the value of $f_{\mathrm{c}}$. 
$f_{c}=L$

Sequences like those in Figure 2 with $f$ are well known although there has been more published on silver than gold. We repeat it here to contrast with the equivalent meso-gold's $R$ versus $f$ data that follows. A good indicator of 2-D character is voids spanning the film cross-section and no internal voids isolated within the film bulk. Note we have taken the area fill factor $f$ in 2-D as linear in mass deposited and hence in deposition time $t$ as proven in reference [15], and the critical deposition time is $t=t_{\mathrm{c}}$. Another approach is to take the coverage in a randomly deposited particle model [18] in 2-D. Generalizing these equations, the time dependence of $f$ is no longer linear in time $t$ but instead obeys

$f(t)=1-\left[1-f_{c}\right]^{t / t_{c}}$

We have also analyzed our resistance data using such a model for coverage $f$ versus time and find a much poorer fit to equations $(1 \mathrm{a}, \mathrm{b})$ than in Figure 2. Equation (3) does not allow for diffusion after deposition, while gold is actually very mobile. Thus, while it might work for refractory metals, it is unlikely to be suited to growing gold or silver layers.

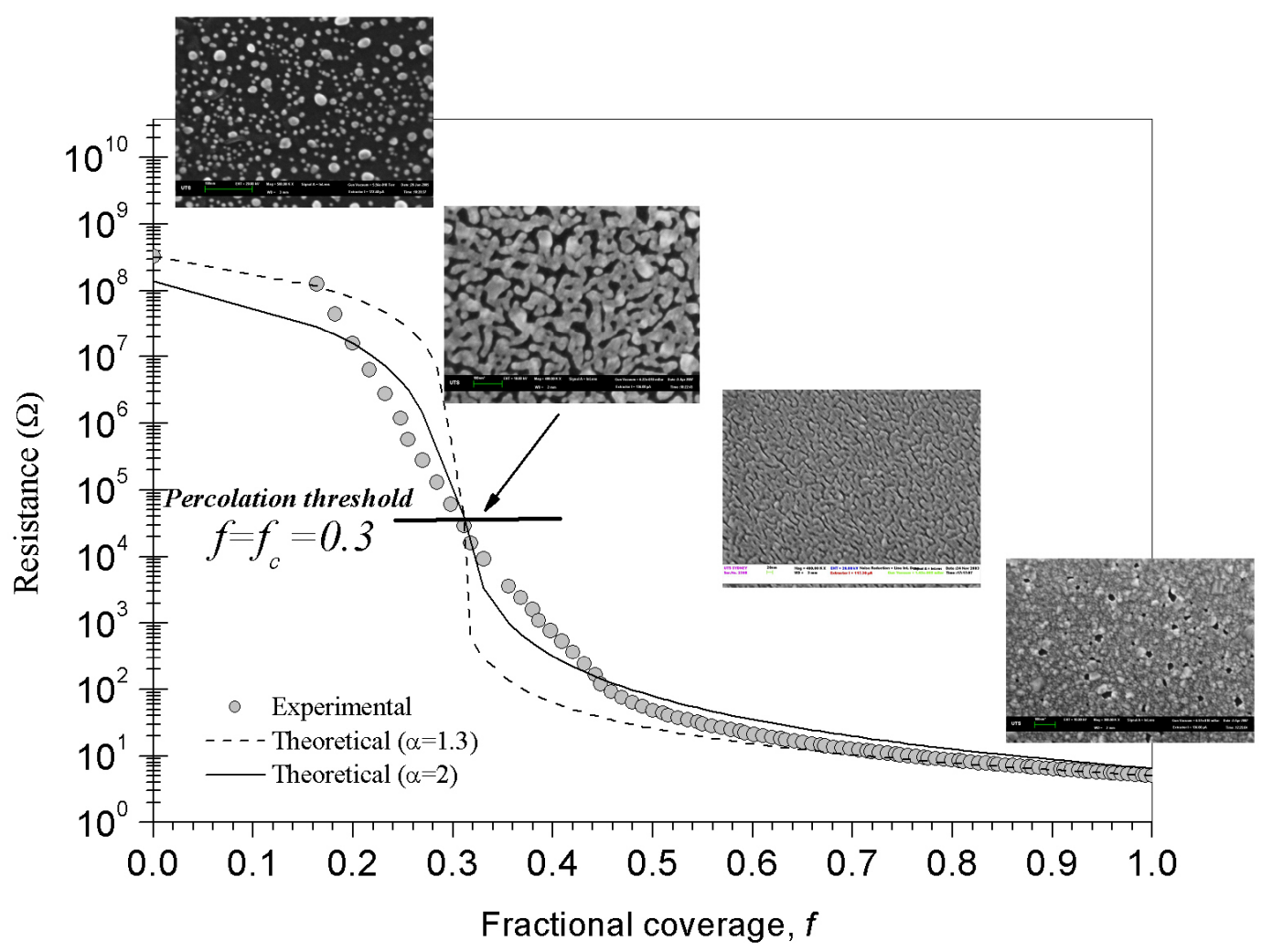

Figure 2: Measured resistance of a thin gold layer through the percolation threshold as a function of fractional coverage. The theoretical plots are for $\alpha=1.3$ and $\alpha=2$. Micrographs demonstrate the structure sequence with fractional coverage.

We now contrast mesoporous gold. For the thicker mesoporous systems $f$ is difficult to estimate from micrographs because most voids are internal. An analysis of the combination of optical, plasmonic and dc responses indirectly provides values for both $f$ and $f_{c}$, and we use these here in analyzing dc data. Using results of effective medium analyses 
of optical and plasmonic data [5] yields $f$ and the effective depolarisation parameter $L$. $L$ in equation (2) then leads to $f_{\mathrm{c}}$ for a given structure. $L$ in these EMA's is actually linked to the array structure rather than individual particle shape. Connectivity $z$ and the hyper-dimension $d$ satisfy $1 / L=z / 2$ and hence $1 / L=d$.

The resulting dc properties are quite interesting, but more complex than those in the thin layer systems. We have found critical concentrations of gold, as low as 0.07 and up to 0.3 in one class of meso-gold films and $f_{\mathrm{c}} \sim 0$ in another $[4,5]$. The actual films that lead to these values always have higher $f$ values than $f_{\mathrm{c}}$, since we cannot span the percolation threshold in these very porous 3-D systems, so equation (1a) only is thus of interest. They would in practice collapse, possibly into a 2-D structure with quite different percolation properties. (It is of interest to note we have actually achieved such an effect by heating). In Figure 3 we plot theoretical values of resistance as a function of $f$ using experimental optical $L$ values for two different meso-gold films, which have also been electrically characterized at one $f$ value. We have used this $R(f)$ data point and the $R_{\text {metal }}$ limit to estimate $\alpha$ and $A$ as needed to create these plots. The value of $\alpha$ given in the caption is close to 1.0 in both. It was at first expected to be near 2 for 3-D structures. This may be due to the hyper-dimensionality influence on the critical exponent for electrical response, but further work is needed, as small changes in $\alpha$ do not have a large effect. In addition we have neglected changes in the electron relaxation rate as found in optical measurements [4]. $\alpha=1$ is the mean field result so this may mean we are not close enough to $f_{\mathrm{c}}$ to extract $\alpha$.

Complexity is compounded because we cannot change $f$ without also changing $L$ and $f_{\mathrm{c}}$ in meso-gold until quite large thicknesses, even for the same preparation conditions. Topology and connectivity is evolving with thickness. Obtaining various $f$ at one $L$ would allow an analogous plot to Figure 2. It may be possible in future with a better understanding of how structures change with thickness and initial deposition conditions.

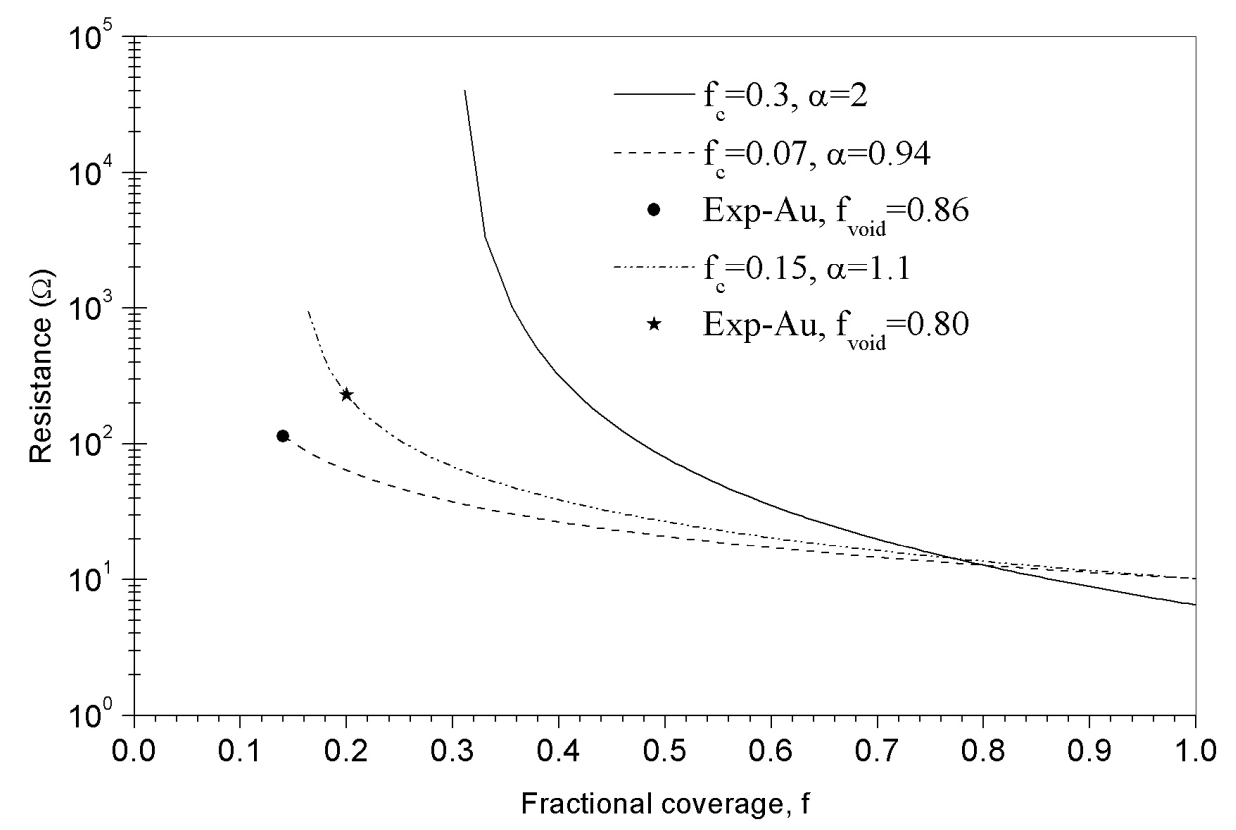

Figure 3: Resistance above the percolation threshold $f_{c}$ for two mesoporous gold systems and thin gold film.

\subsection{Plasmonic phenomena}

\subsubsection{Simulated optical properties}

The results of the numerical simulations of extinction coefficient Qext are shown in Figures 4(a) and 4(b). Extinction coefficient in these layers is predominantly due to absorption, not to scattering. In Figure4(a) we show a selection of 15 nm thick "targets" obtained when a particular geometric design was rendered to increasingly finer lattices. The DDA 
dipole volumes vary from $15 \mathrm{~nm}^{3}$ to $1.13 \mathrm{~nm}^{3}$ for the targets shown. It is evident that targets with large dipoles produce numerical instabilities in the calculation, and that only those simulations with dipoles of less than about $2 \mathrm{~nm} 3$ are suitable. The very small dipoles needed here are the consequence of the very large surface to volume ratio of the mesoporous targets. (In fact, our experience has shown that stable computations are possible in "solid" targets such as spheres or nanorods with dipole volumes as high as $20 \mathrm{~nm}^{3}$ ). In Figure 4(b) we show the results of three separate Monte Carlo simulations of a sponge $40 \mathrm{~nm}$ thick, once again at $24 \%$ density. Although the extinction spectra between targets differ slightly, due either to genuine geometric effects or possibly small numerical instabilities, the overall trends are the same. The $40 \mathrm{~nm}$ thick targets naturally have greater extinction efficiency than the $15 \mathrm{~nm}$ thick ones, however, it is clear that the extinction peak for the latter is considerably more red-shifted. The rate of convergence of DDSCAT calculations on metals such as gold or silver deteriorates markedly for wavelengths greater than about $1000 \mathrm{~nm}$, which prevented examination deeper into the NIR. This red shift in the thinner simulated layers agrees with our experimental optical data on mesoporous films that follow. A red shift there as thickness drops is associated with a lower effective plasma frequency and hence a shift of plasmonic based extinction to longer wavelengths.

(a)

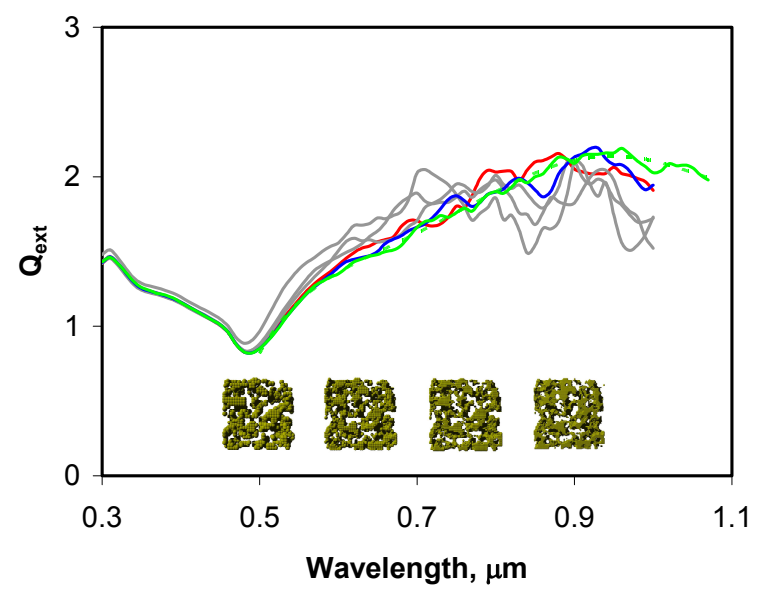

(b)

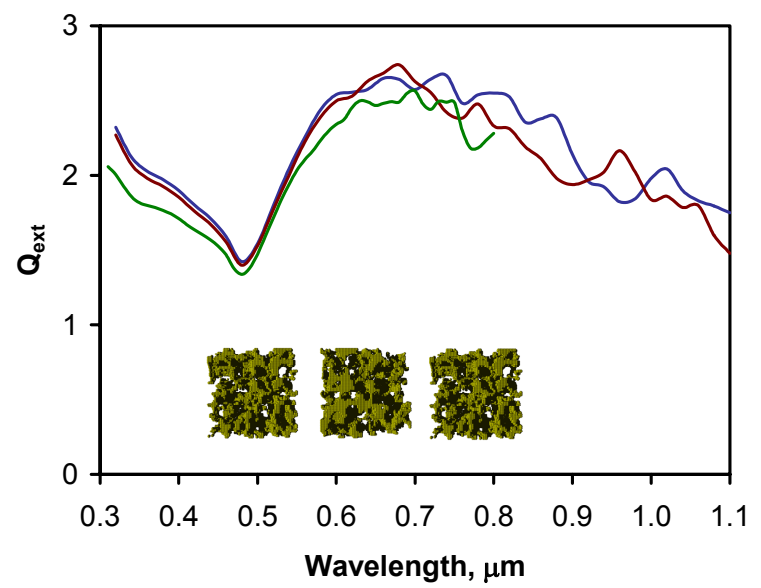

Figure 4: Simulations of the optical extinction efficiency of a slab of mesoporous gold. (a) A slab measuring $100 \times 100 \times 15$ $\mathrm{nm}$, with an average density of $24 \%$ gold, rendered with dipole volumes varying from $14 \mathrm{~nm}^{3}$ to $1.1 \mathrm{~nm}^{3}$. (b) Three randomly generated slabs measuring $100 \times 100 \times 40 \mathrm{~nm}$, with an average density of $24 \%$ gold, rendered at dipole volumes in the range of 5 to $8 \mathrm{~nm}^{3}$. 


\subsubsection{Experimental optical properties}

Here we contrast the two distinct topologies and films discussed in the resistive Section 3.1, in terms of their experimental plasmonic responses, and also see which is most closely linked optically to the simulated thin film void array just outlined in 3.2.1. We present the data for films analyzed electrically. To make comparison easier to extinction models, spectral absorptance plots are shown for three of the thin gold layers in Figure 5, and in Figure 6 for the two mesoporous layers with $f_{c}=0.07, f=0.14$, and with $f_{c}=0.15, f=0.20$ plus we add one plot from the class of films with $f_{c}$ $=0$.

The key distinctions link to plasmonic effects. Differences in NIR absorptance profiles in Figure 5 are broad but clear. They link to averages over local surface plasmon resonance effects associated with the film-crossing voids [2,3,9]. Meso data in Figure 6 has the normal dip for gold nanoparticles around 500nm plus an NIR peak which red shifts in the thinner layer (c.f. Figure 4). This mesostructured experimental absorptance data is in better qualitative agreement with the simulated meso-layer extinction data in Figure 4, than the thin gold layers in Figure 5. We can attribute the meso-data to effective or homogenised bulk properties since an effective Drude model gives a good fit to data [4] . This also means the meso gold is in effect plasmonic and has an effective plasma frequency $\omega_{p}$. As expected $\omega_{p}$ is well below that of dense gold, but is also influenced by a homogenised effective mass not just gold carrier dilution [5]. It is the effective mass $m^{*}$ that links to the dimensionality found in our de studies since $m^{*}$ links directly to $f_{c}$, Thus in meso-porous gold the effective plasma frequency depends on the percolation threshold and on the hyper-dimensionality as well as $f$.

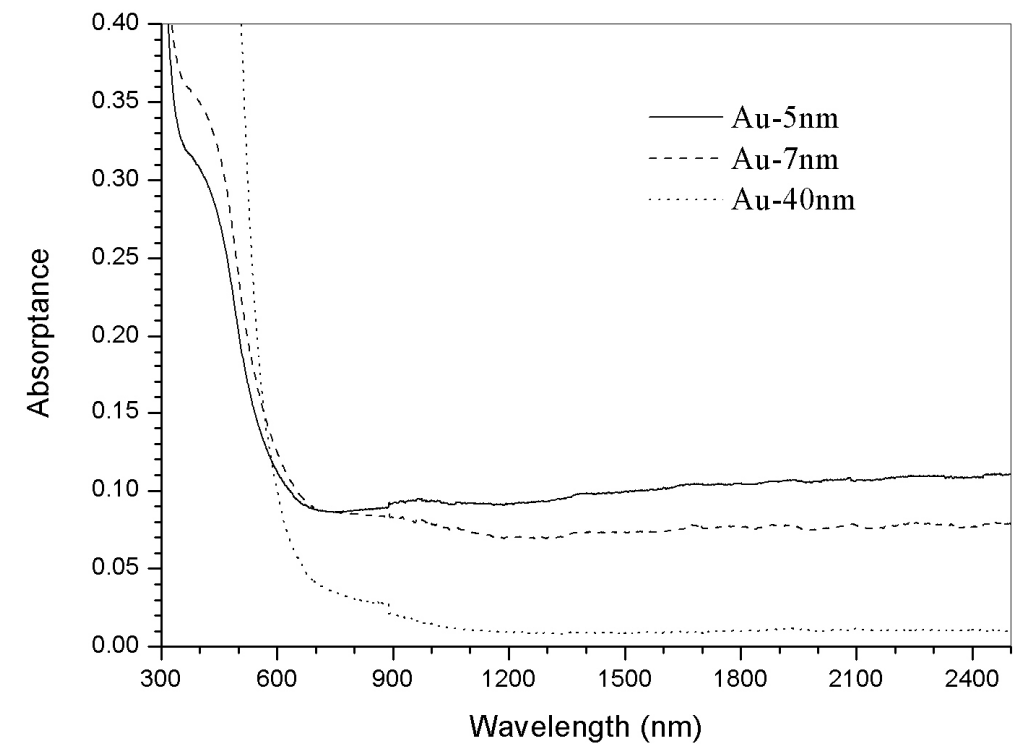

Figure 5: Spectral absorption of thin gold films on glass substrate. The $40 \mathrm{~nm}$ layer has no voids. 


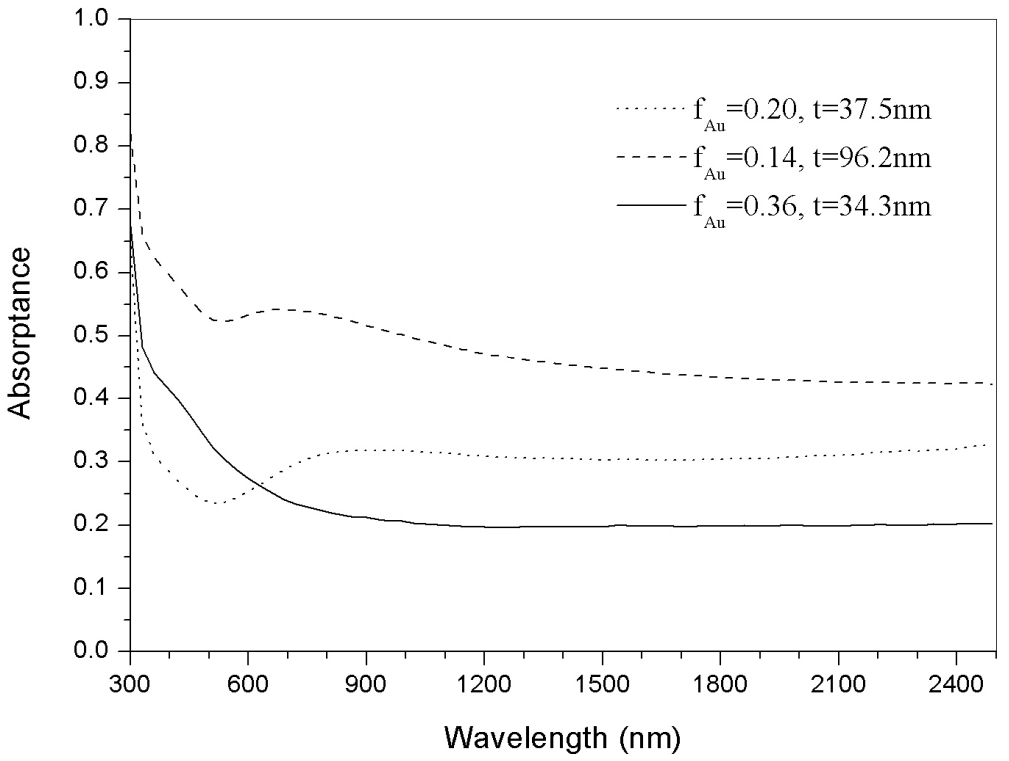

Figure 6: Spectral absorption of mesoporous gold films. Samples with $f=0.2$, and $f=0.14$ deposited without ion-assist while the sample with $f=0.36$ was deposited with ion-assist.

The thin film porous gold in Figure 5, in contrast, cannot be modeled as a simple bulk plasmonic system. Nor can it be modeled meaningfully as a standard thin film of known thickness and effective optical constants. This is because the spectral features involve both localized and de-localized surface plasmons [19] and their associated evanescent fields at the gold-air interface. These interface effects modify the absorptance and reflectance [20] in a manner determined by the actual surface nanostructure, in particular its spatial frequency components. These surface plasmonic responses should also link to the $f_{c}$ and $f$ values via surface statistics of location of gold and voids. Voids that cross these layers enhance the coupling of these SP interface effects across the thin metal layer and add absorption not found in usual thin film models unless physically incorrect optical constants are used. Thus in terms of plasmonic effects and their link to resistive behavior as a function of $\mathrm{f}$ we are looking at two distinct phenomena in the two types of films studied here. The connectivity or dimensionality plays a key role in both. In meso-gold it determines effective bulk plasmonic response while in very thin vacuum deposited gold-void films it determines along with film thickness surface plasmon response. Surface plasmons can be neglected in the mesoporous layers [21].

\section{CONCLUSION}

Electrical and plasmonic response in gold containing nanoscale pores is very sensitive to dimensionality $d$, Values of $d$ from 3 to over 10 and critical percolation with as little as 7\% gold, have been indicated by experimental data on mesoporous gold. Thin granular gold films have quite different percolation values with $f_{c}=0.3$, which is consistent with the directly observed 2-D morphology, provided it is considered as a "Swiss-cheese" topology rather than a particle cluster system. Simulations of optical extinction by 3-D "Swiss-cheese" type gold-void layers with very high void content are in qualitative agreement with observations on mesoporous gold. 


\section{ACKNOWLEDGEMENTS}

We thank Geoff McCredie for assistance with optical characterization and Ric Wuhrer for help with electron microscopy.

\section{REFERENCES}

1. A.I. Maaroof, and B.L. Evans,"'Onset of electrical conduction in Pt and Ni films",J. Appl. Phys. 76(2) 10471054(1994).

2. K.Seal, M.A. Nelson, Z. Charles, D.A. Genov A.K. Sarychev and V.M. Shalaev, "Metal coverage dependence of local optical properties of semicontinuous metal films" J. Modern Optics, 49, 2413-2435 (2002)

3. D.A. Genov, A.K. Sarychev and V.M. Shalaev and A. Wei, "Resonant field enhancements from metal nanoparticle arrays", Nano Letters, 4, 153-158 (2004)

4. G.B. Smith, A. Maaroof and A. Gentle "Homogenized Lorentz-Drude optical response in highly nanoporous conducting gold layers produced by de-alloying”, Optics Communications, 271, 263-268 (2007).

5. G. B. Smith A.R. Gentle and A.I. Maaroof "Metal insulator composites which act optically like homogeneous conductors", J. Nanophotonics (SPIE Digital Library) 1, 013507 (2007).

6. G.B.Smith, G.A.Niklasson, J.S.E.M.Svensson, C.G.Granqvist "Noble -metal- based transparent infra-red reflectors: Experiments and Theoretical analyses for very thin gold films", J.Appl.Phys., 59,571-581,(1986)

7. A.I. Maaroof and G.B. Smith,"Effective optical constants of nanostructured silver films and impact of an insulating overlayer", Thin Solid Films, 485, 198 - 206 (2005)

8. S. Gresillon, L. Aigonouy, A.C. Boccara, and J.C. Rivoal, X. Quelin, C. Desmarest, and P. Gadenne, V. A. Shubin, A. K. Sarychev, and V. M. Shalaev, "Experimental observation of localized optical excitations in random metaldielectric films', Phys. Rev. Lett. 82, 4520-4523(1999).

9. D.A. Genov, K.Seal, M.A. Nelson, A.K. Sarychev, Z.C. Ying and V.A. Shalaev, " Local field distribution in randsom metal-dielectric films; theory and experiment" Physica B, 228-231 (2003).

10. "Coupled multipolar interactions in clusters of nanoparticles with metal shells",_G.B. Smith and V Pustovik Optical Communications, 211, 1-6, 197-204 (2002).

11. W. Xia and M.F. Thorpe, "Percolation properties of random ellipses", Physical Review A, 38, 2650-2656 (1988).

12. A.I. Maaroof, M.B. Cortie, A Gentle and G.B. Smith "Mesoporous gold sponge as a prototype meta-material", Physica B ,394, 167 - 170 (2007).

13. A.I. Maaroof, M.B. Cortie, and G.B. Smith, "Optical Properties of mesoporous gold films", J. Optics A. Pure and Applied Optics, 7, 1-7 (2005).

14. B. T. Draine, and P. J. Flatau, "Discrete-dipole approximation for scattering calculations", J. Opt. Soc. Am. A 11, 1491-1499 (1994).

15. Shi Xu, B. L. Evans, David I. Flynn and Cao En ,"The study of island growth of ion beam sputtered metal films by digital image processing", 238, 54-61(1994).

16. G. Ord, B. Payandeh and M. Roberts, "Analytic solution of the block-cluster theory of site and bond percolation in arbitrary dimension", Phys. Rev. B, 37, 467-471 (1988).

17. G. B. Smith "Dielectric constants for mixed media", J.Phys. D.(App.Phys.) 10,139-42,(1977).

18. D.J. Bergman and D. Stroud, Solid State Physics, Vol. 46, pp147, Academic Press, 1992.

19. K.Seal, A.K.Sraychev, H. Noh, V.M. Shalaev, Z.C. Ying . X. Zhang and H. Cao, "Coexistence of localised and delocalised surface plasmon modes in percolating metal films" Phys. Rev. Letts., 97, 206103 1-4(2006).

20. Y. Tachibana, Y. Kusunoki, and H. Ohsaki, Vacuum, 74, 555-559 (2004).

21. A.I Maaroof, A. Gentle, G.B. Smith and M.B. Cortie "Bulk and surface plasmons in highly nanoporous gold films" J. Phys. D: Appl. Phys.(in press,2007). 